\title{
Iconic turn et critique du paradigme langagier
}

\author{
Par MAUD HAGELSTEIN \\ FNRS - Université de Liège
}

Comment appréhender le problème de la légalité des significations dans le champ non verbal du visuel ? Tel est l'exercice auquel s'essaie le théoricien de l'image allemand Gottfried Boehm, soucieux de dégager les principes formels structurant l'émergence de significations dans le domaine du visuel, tout en tenant à distance l'usage de la grammaire prédicative pour la compréhension des images. Sa proposition s'inscrit dans le cadre des débats autour de ce que l'on appelle - dans le domaine de la théorie de l'image actuelle - l'iconic turn (le "tournant iconique »/ ikonische Wendung). À bien des égards, l'iconic turn est lié à une réflexion large sur l'héritage de l'iconologie, cette discipline d'interprétation des images inaugurée en 1912 par le théoricien de l'art Aby Warburg (1866-1929), nourrie par la philosophie des formes symboliques de Ernst Cassirer (1874-1945), et systématisée par Erwin Panofsky (1892-1968) dans ses Essais d'iconologie (1939), notamment ${ }^{1}$. Visant l'interprétation du contenu symbolique des images, la méthode iconologique concevait l'art comme l'un des domaines essentiels de production de sens, une «forme symbolique » privilégiée, pour reprendre la terminologie cassirérienne ${ }^{2}$. Le célèbre tableau synthétique repris en introduction aux Essais d'iconologie montre comment procède l'iconologie, par

1 E. Panofsky, Studies In Iconology: Humanistic Themes In The Art Of The Renaissance (1939), traduit de l'anglais par C. Herbette et B. Teyssèdre : Essais d'iconologie : thèmes humanistes dans l'art de la Renaissance, présenté et annoté par B. Teyssèdre, Paris, Gallimard, 1967. Pour le texte qui inaugure chez Aby Warburg l'usage du terme « iconologie », voir A. Warburg, " Italienische Kunst und internationale Astrologie im Palazzo Schifanoia zu Ferrara », Gesammelte Schriften, Band I.2. Die Erneuerung der heidnischen Antike, Berlin, Akademie Verlag, 1912.

2 Voir principalement l'introduction au projet en trois volumes de E. Cassirer, Philosophie der symbolischen Formen. I. Die Sprache, Gesammelte Werke. Bd. XI, Hamburg, Felix Meiner, 2001. Trad. française d'O. Hansen-Love et J. Lacoste : La philosophie des formes symboliques (1. Le langage), Paris, Minuit, 1972. 
dégagement progressif de couches de significations. S'il a construit sa méthode en dialogue avec la philosophie, Panofsky n'a pas pour autant intégré au cahier des charges de l'iconologie l'ambition de dégager systématiquement les principes formels conditionnant la possibilité même de la signification - raison pour laquelle cette discipline n'est pas à proprement parler philosophique. L'iconologie est exégétique ; elle offre les outils nécessaires à une interprétation précise du contenu des œuvres.

Pour les principaux protagonistes des débats repris sous le vocabulaire $\mathrm{du}$ «tournant iconique », Panofsky aurait commis l'erreur regrettable de soumettre les images à l'ordre tout puissant du langage (à partir de la fin des années 1980, il devra subir et adosser pour cette raison le rôle d'épouvantail de la théorie de l'image contemporaine). L'historien de l'art aurait réduit la réception des œuvres d'art à l'établissement raisonné d'un vocabulaire des formes et à la description des règles combinatoires, négligeant les apports de l'expérience perceptive. Et effectivement, à suivre ses analyses, on doit bien constater que Panofsky incite lui-même à comprendre le visuel sur le modèle $\mathrm{du}$ langage ${ }^{1}$. On trouve dans ses textes l'idée d'une mise en rapport syntaxique des différents éléments picturaux de l'œuvre, qui tirent leur sens de leur connexion les uns avec les autres. Panofsky étudie les modes d'assemblage des motifs dans un programme iconographique, convaincu que l'œuvre d'art fonctionne comme un système où la syntaxe a sa place. Il parle par exemple de "ponctuation » à l'égard de certains éléments formels qui fonctionnent comme indices d'un bon ordre de lecture ${ }^{2}$. Telle que Panofsky l'envisage, l'iconologie ne se réduit pas à la seule identification des figures et des thèmes, comme on veut parfois le croire, mais elle vise un décodage global de la répartition des éléments signifiants.

La critique la plus basique (et néanmoins communément partagée) adressée aujourd'hui à Panofsky consiste à dire et à redire inlassablement qu'il semble avoir, au moment de l'institution de cette méthode, écarté la plupart du temps les préoccupations formelles, tout ce qui concerne les qualités proprement plastiques et picturales des œuvres, pour se concentrer avec plus d'insistance sur les éléments de contenu et analyser au mieux les liens de l'œuvre d'art aux significations. En héritière directe et parfois ingrate des idées de Panofsky, la théorie de l'image allemande est partie de là pour développer des perspectives nouvelles. En effet, tout en cherchant à en

\footnotetext{
${ }^{1}$ Voir les excellents développements d'A. Rieber, Art, Histoire et signification. Un essai d'épistémologie d'histoire de l'art autour de l'iconologie d'Erwin Panofsky, Paris, L'Harmattan, 2012.

${ }^{2}$ Id.
} 
actualiser les principes, les protagonistes de la Bildwissenschaft ont adressé à l'iconologie panofskienne des critiques parfois féroces, visant principalement sa tendance présumée à assécher l'œuvre de sa matérialité pour n'en faire ressortir que des significations extérieures à elle et qui lui préexistent c'est-à-dire concrètement pour faire émerger le (pré-)texte gouvernant la réalisation des œuvres. Depuis la fin des années 1980, de nombreuses tentatives visent à donner au concept d'iconologie un sens actuel, l'iconologie désignant - en ce sens élargi qui ne trahit cependant pas les intentions de ses pionniers - l'étude transdisciplinaire des logiques iconiques ${ }^{1}$. On continue aujourd'hui plus que jamais à poser la question de l'interprétation des éléments visuels de nos cultures et à réclamer une discipline transversale capable de décrire la mécanique des images. Les recherches reprises sous la catégorie Bildwissenschaft explorent en ce sens des domaines variés de l'expression et de l'expérience visuelle (art, science, médias, politique) ${ }^{2}$.

\section{Changement de paradigme : le tournant iconique}

Bien connu des spécialistes de l'image, le débat autour de l'iconic turn, caricatural dans ses premières formulations mais s'assouplissant au fur et à mesure de ses actualisations, mérite donc d'être repris et analysé comme débat programmatique pour la théorie de l'image ${ }^{3}$. Pour le dire une première

\footnotetext{
${ }^{1}$ Sans s'expliquer sur leur choix, « la plupart » de ces auteurs préfèrent l'idée de logique à celle de grammaire.

${ }^{2}$ Lire par exemple H. Belting, Das Ende der Kunstgeschichte : eine Revision nach zehn Jahren, München, C.H.Beck Verlag, 2002 ; H. Bredekamp (co-éd.), Aby Warburg. Akten des internationalen Symposions, Berlin, Akademie, 1990 ; H. Bredekamp, «Ex nihilo : Panofsky's Habilitation » in Polyanthea : essays on art and literature in honor of William Sebastian Heckscher, The Hage, Van der Heijden, 1993 ; T. Lenain, H. Locher, A. Pinotti, M. Rampley, C. Schoell-Glass, K. Zijlmans (éds. ), Art History and Visual Studies in Europe, Leiden, Brill, 2012 ; R. Recht, "L'historien de l'art est-il naïf ? Remarques sur l'actualité de Panofsky », in M. Warnke (éd.), Relire Panofsky, Paris, Éditions du Musée du Louvres, 2008 ; K. Sachs-Hombach, Bildwissenschaft zwischen Reflexion und Anwendung, Köln, Halem Verlag, 2005 ; K. Sachs-Hombach (éd.), Bildtheorien. Anthropologische und kulturelle Grundlagen des Visualistic Turn, Frankfurt/Main, Suhrkamp, 2009.

${ }^{3}$ Voir à ce propos le numéro de la revue bilingue Trivium (Revue franco-allemande de sciences humaines et sociales - Deutsch-französische Zeitschrift für Geistes- und Sozialwissenschaften) consacré à ces questions : "Iconic Turn » et réflexion sociétale, sous la direction de G. Didi-Huberman et B. Stiegler (2008). URL : https://trivium.revues.org/223
} 
fois sans nuance, les défenseurs du tournant iconique pensent renverser la domination du paradigme langagier pour l'interprétation des images. Plutôt que de se concentrer exclusivement sur l'étude des contenus symboliques, il importe à leurs yeux de se concentrer sur ce qui fait la spécificité intrinsèque de l'image et de montrer comment sa matérialité affecte directement la production du sens ${ }^{1}$. On observe par ailleurs et par conséquent dans l'analyse iconologique des œuvres, notamment photographiques, un élargissement des éléments de l'image susceptibles d'être porteurs de signification: cadrages maladroits, bougés, ombres, éléments aniconiques (taches), flous, saturations, etc., importent autant que l'identification des seules figures ${ }^{2}$. L'image déploie ses effets sur un mode propre sans que le secours du langage soit forcément nécessaire, en tout cas dans un premier temps. Il y aurait des éléments formels concrets en excès par rapport au message que porte l'image, un surplus sensible intraduisible. Parmi les principaux acteurs de la Bildwissenschaft, le philosophe Gottfried Boehm rejoint à cet égard ceux qui réclament une version critique renouvelée de l'iconologie qui ne s'appuierait plus sur le paradigme langagier.

Au début des années 1990, on assiste en effet à l'émergence d'un nouveau paradigme énoncé - de manière coordonnée mais apparemment sans concertation préalable - par l'américain William J. Thomas Mitchell (qui a forgé en 1992 le concept de Pictorial Turn $^{3}$ ) et par l'allemand

${ }^{1}$ L'image elle-même est cela : une entité matérielle générant du sens. Cf. Boehm : «Dans le sens d'une compréhension élémentaire, on pourra le [= le pouvoir des images] ramener à un substrat matériel. Or, il est certain que les images ne se réduisent pas à la matière. Sur des surfaces, dans la poussière de la couleur, dans la pierre, sur du bois ou de la toile, sur des supports photosensibles ou sur des écrans digitaux, il y a toujours quelque chose d'autre qui se montre : une vue, un spectacle, un sens - une image en somme » (G. Boehm, " Par-delà le langage ? Remarques sur la logique des images », Trivium [En ligne], 1 | 2008, mis en ligne le 08 avril 2008, consulté le 27 janvier 2015. URL : http://trivium.revues.org/252). Ceci dit, on ne voit pas en quoi cette définition (une entité matérielle générant du sens) constitue la spécificité de l'image visuelle. Quid des sons, de la musique, par exemple?

${ }^{2}$ Relire par exemples les analyses de G. Didi-Huberman, Images malgré tout, Paris, Minuit, 2004. À partir de quatre photographies prises dans un contexte concentrationnaire, au péril de leur vie, par des membres du Sonderkommando, Didi-Huberman montre comment des éléments formels apparemment anecdotiques peuvent s'avérer essentiels pour la compréhension de la situation d'urgence dans laquelle ont été pris ces clichés.

${ }^{3}$ W. J. T. Mitchell, « The pictorial turn », Picture Theory. Essays on Verbal and Visual Representation, Chicago, University of Chicago Press, 1994, p. 11-35. Étude publiée en mars 1992 dans Artforum. 
Gottfried Boehm (qui a développé en 1994 l'idée d'Iconic Turn, ou ikonische Wendung, dans l'ouvrage Was ist ein Bild $\left.{ }^{1}\right)$. En réponse aux arguments de la célèbre anthologie dirigée par Richard Rorty en 1967 (The Linguistic Turn), ouvrage qui entendait rééquilibrer le programme intellectuel de la deuxième moitié du $\mathrm{XX}^{\mathrm{e}}$ siècle en libérant la philosophie de son emprisonnement dans la visualité, Mitchell et Boehm exigent un nouveau revirement : il s'agit d'essayer de comprendre de quelle manière les images organisent les significations, de définir ce qu'est le «logos iconique», et en quoi une « proposition visuelle » diffère d'une « proposition langagière ». Pourquoi le « sens visuel» d'une œuvre ou d'une image ne se laisse-t-il pas facilement « transcoder » dans le langage (en tout cas pas sans perte) ?

Concernant la proposition théorique de Gottfried Boehm, on peut se référer notamment au texte intitulé «Jenseits der Sprache ? Anmerkungen zur Logik der Bilder » ("Par-delà le langage ? Commentaires sur la logique des images ») $)^{2}$, ainsi qu'à un texte synthétique intitulé : «Ce qui se montre. De la différence iconique $»^{3}$. Ce dernier texte reprend une intervention au Collège international de philosophie (séminaire 2007-2008) basée sur des arguments déjà développés ailleurs, notamment dans le texte d'ouverture de l'ouvrage Was ist ein Bild?, intitulé «Die Wiederkehr der Bilder» (« le retour des images ») et dans l'ouvrage Theorie des Bildes («Théorie de l'image », 1999). Philosophe et historien de l'art, Gottfried Boehm est Professeur à l'Université de Bâle et fondateur du centre de recherche international eikones - Bildkritik. Bien qu'il ne discute pas directement ces auteurs, on trouve dans ses textes des références très nombreuses à la psychologie de la forme (Gestalt Theorie), à Wittgenstein et à la phénoménologie (en particulier celle de Merleau-Ponty: l'ouvrage Was ist ein Bild? comprend d'ailleurs, juste après son propre texte, une traduction en allemand de « Le doute de Cézanne »).

Boehm fait partie de ceux qui ont appelé de leurs vœux un tournant iconique (ikonische Wendung), selon la formule qu'il a forgé lui-même en 1994, convaincu de la nécessité — sur laquelle il insiste dans pratiquement

${ }^{1}$ G. Boehm (éd.), Was ist ein Bild?, München, 1994. Cf. sur ces questions voir B. Stiegler, «"Iconic Turn" et réflexion sociétale », Trivium [En ligne], $1 \mid 2008$, mis en ligne le 08 avril 2008, consulté le 22 janvier 2015. URL : http://trivium.revues.org/308

${ }^{2}$ Publié à l'origine dans : C. Maar, H. Burda (éds.), Iconic Turn. Die Neue Macht der Bilder, Köln, Dumont, 2004.

${ }^{3}$ G. Boehm, «Par-delà le langage ? Remarques sur la logique des images », op. cit.; G. Boehm, « Ce qui se montre. De la différence iconique », Penser l'image, E. Alloa (éd.), Presses du réel, 2010, p. 27-47. 
tous ses textes - de comprendre la logique non discursive des images : «Par logique, nous entendons l'engendrement cohérent de sens à partir d'authentiques moyens iconiques. Et pour explication, j'ajoute que cette logique est non prédicative, ce qui veut dire qu'elle n'est pas formée sur le modèle de la proposition ou d'autres formes langagières. Elle n'est pas parlée mais réalisée en étant perçue $»^{1}$. Boehm se réfère pour la compréhension de la logique propre aux images au modèle du performatif - le visuel serait performatif, au sens d'Austin, en tant que ses "énoncés » ne sont pas descriptifs mais qu'ils font ce qu'ils énoncent. En effet, «ce qui est décisif pour que naisse le sens c'est de raviver dans l'image l'acte de voir, qui y est latent $»^{2}$.

La tradition philosophique aurait décrédibilisé durablement l'image en raison de son statut flottant: les théories iconoclastes sont nombreuses qui ont exclu les images du logos "précisément au titre de leur équivocité, le logos finissant par être lui-même réduit à une logique propositionnelle de type langagier. La prédication devient ainsi le modèle de tout sens authentique, permettant d'établir sans équivoques ce qui est et ce qui n'est pas. Les images ne seront raisonnables qu'à condition de participer au langage : un logos iconique reste inconcevable $»^{3}$. Gottfried Boehm se saisit donc de la problématique et revendique l'idée suivante: la logique des images serait une logique de la monstration. Par définition, l'image est l'opération selon laquelle un élément matériel indique un immatériel. L'image fait voir ; elle montre. Pour le dire autrement, l'iconicité (Bildlichkeit), c'est-à-dire le «f faire-image » ou le pouvoir sémio-génétique du visuel, se définirait par son "potentiel déictique », lui-même profondément conditionné par la possibilité, pour un corps vivant, d'être en mouvement dans un espace visible. Et — de surcroît - le propre des images artistiques serait de nous donner à voir cette logique de l'incarnation du sens dans l'espace visuel et moteur. Boehm va même jusqu'à supposer - la radicalité de son tournant en dépend - que la logique de la monstration basée sur la capacité du corps à indiquer des directions détermine aussi l'exercice du langage (au sens où il y aurait des " évidences intuitives et iconiques qui

${ }^{1}$ G. Boehm, « Par-delà le langage ? Remarques sur la logique des images », op. cit. Dans cette citation, on peut se demander ce que recouvre l'épithète " cohérent", expression laissant supposer qu'un engendrement incohérent de sens serait possible mais ne mériterait pas le statut de logique (de quoi parlerait-on dans ce cas ?). La cohérence ne prend-elle pas ici le relais de ce que la structure langagière visait? ${ }^{2} I d$.

${ }^{3}$ G. Boehm, « Ce qui se montre. De la différence iconique », op. cit., p. 34. 
aident la langue à réaliser ses possibilités ${ }^{1}$ ). L'iconicité ne serait pas à considérer comme un registre parmi d'autres mais comme le « fond déictique de toute expression », du phénomène expressif en général, qu'il soit verbal ou non. Avec cette thèse, et ceci nous autorise probablement une première réserve, Boehm suppose l'anhistoricité de la logique des images, qui échapperait aux déterminations du régime historique des signes conditionnant l'expression. Comme si notre position singulière au sein de l'histoire du signe n'avait aucune influence sur la généralité du phénomène expressif propre à l'image.

Le langage lui-même serait soumis à cette incarnation originaire dans l'espace visuel et moteur: comme l'a montré le linguiste allemand Karl Bühler, les déictiques jouent un rôle essentiel pour le langage. Il y a un « champ monstratif» du langage que les particules monstratives (ça, ici, là) - par exemple - manifestent. Pour Boehm, les images supplantent par moment le langage en tant qu'elle font apparaître - «par nature » en quelque sorte - le champ monstratif originaire dont dépend le langage luimême. Il faut bien avouer que, dans les textes convoqués ici en tout cas, ces

\footnotetext{
${ }^{1}$ Wittgenstein lui-même est chez Boehm à l'origine de cette réflexion : « C'est tout particulièrement Wittgenstein qui a montré que la plausibilité des concepts langagiers repose sur le caractère intuitif et imagé de la langue du quotidien. C'est ainsi qu'il s'est par exemple interrogé, dans les Recherches philosophiques, sur le concept de "jeu" qui apparaît en tant que jeu de dames, de cartes, de balle, de combat, de football, de handball etc. Qu'est-ce qui fonde cette identité, qu'est-ce qui est commun à tous de telle sorte que l'on puisse parler au singulier d'“un" jeu ? "[ [...] si tu les regardes, tu ne verras certes pas quelque chose qui serait commun à tous, mais tu verras des similitudes, des parentés, et même toute une série. Alors, encore une fois: ne pense pas, mais regarde !" (Ludwig Wittgenstein, Philosophische Untersuchungen, Werkausgabe, t. 1, Frankfurt/Main, 1984, paragraphe 66, p. 277). Wittgenstein sollicite par conséquent l'imagination ("Regarde !") et l'analogie des concepts pour entreprendre la fondation des savoirs; il ramène la proposition à des contextes flous ou, plus précisément - pour le dire dans les termes de son contemporain autrichien Robert Musil —, le sens du réel au sens du possible; les deux n'étant que des aspects différents du même scepticisme langagier. Le "lit du fleuve des pensées" ["Flussbett der Gedanken"] n'est pas lui-même de nature langagière ; la critique du langage restitue aux images qui se trouvent en nous et dans le langage quotidien leur droit fondateur et leur fonction indicatrice. Ce sont des évidences intuitives et iconiques qui aident la langue à réaliser ses possibilités. Ici apparaît un déplacement qui fait époque : le logos cesse de dominer la potentialité de l'image pour concéder sa dépendance par rapport à elle. L'image trouve accès au cercle intérieur de la théorie, auquel incombe la fondation de la connaissance. » (G. Boehm, « Par-delà le langage ? Remarques sur la logique des images », op. cit.).
} 
affirmations sont peu argumentées. Boehm évoque par exemple - mais sans approfondir la référence - la recherche en neurosciences qui aurait montré que « le discours verbal et le discours gestuel sont commandés par les mêmes régions cérébrales ». Convaincu de l'autonomie du domaine visuel à l'égard du langage verbal (non seulement son autonomie mais aussi sa préséance), Boehm se donne alors pour projet de reconsidérer la capacité des images à générer du sens sans s'adosser à des significations déjà existantes qu'elles ne feraient qu'énoncer à nouveau, leur capacité à produire du sens qui serait directement dépendant de leur matérialité.

Il s'agirait donc de reconsidérer la fonction intransitive des images. Certes, personne n'entend nier que les images rendent d'excellentes «prestations de service » lorsqu'elles illustrent des motifs qu'elles ne génèrent pas elles-mêmes, mais elles sont aussi sources de significations propres ${ }^{1}$. Reste à comprendre comment s'organise la production de ces significations. Boehm reprend des exemples bien connus: les taches aléatoires («macchie») dispersées sur un mur décrépi que Léonard de Vinci dans son Traité de peinture conseille à ses élèves aspirants peintres d'observer jusqu'à ce que des figures apparaissent, ou les images de Warhol inspirées des tests de Rorschach. Autrement dit: autant de représentations "plurivoques (qui) donnent lieu aux interprétations les plus variées $»^{2}$. Ces exemples - que Gottfried Boehm ne se prive pas de reprendre dans d'autres textes montrent non seulement «l'entrainement imaginatif» auquel se plie volontiers l'artiste, mais exhibent la faculté qu'a le regard humain de percevoir des images, c'est-à-dire de percevoir autre chose que de simples objets matériels. Ou plutôt : de s'appuyer sur la matérialité pour construire du sens à partir de là - et sans le secours de significations extérieures que l'on ne ferait que reproduire/illustrer. Boehm entend donc démontrer par cet exemple la force autonome de l'image et sa capacité (plastique/picturale) à exprimer de nouvelles significations grâce aux « possibilités constructives du "voir en tant que" et du "voir dans" ».

${ }^{1}$ « Il n'est nullement étranger aux images de rendre des prestations de service. Elles le font au contraire avec une telle perfection que certains auteurs et le célèbre homme de la rue ne veulent même pas envisager qu'elles aient encore un autre dessein que celui de l'illustration. Mais celui qui souligne par trop fort le texte derrière l'image atterrit invariablement dans la domination du langage, qui méconnait les possibilités de l'image » (Id.).

${ }^{2}$ G. Boehm, « Ce qui se montre. De la différence iconique », op. cit., p. 31. 


\section{La différence iconique : une logique des contrastes}

Le scepticisme langagier que Boehm pense retrouver chez certains philosophes comme Wittgenstein l'amène à investir l'idée d'une "différence iconique » (ikonische Differenz). Si l'on veut trouver — au sein du visuel un principe organisateur structurant, il faut aller voir du côté de cette différenciation essentielle, différenciation opérant à même le matériau sensible, mise en scène par l'image et constitutive du sens. Gottfried Boehm cherche explicitement à sortir l'image des règles de la prédication par la voie d'une théorie de la différenciation : une «pensée avec les yeux » reposerait entièrement sur les contrastes suscités par les images (le contraste entre la forme et le fond constituant apparemment pour Boehm le moteur principal de l'iconicité). Débarrassée de la logique trop mécanique de la prédication qui rassemble des éléments en les associant selon des codes bien établis, la théorie de l'image pourrait envisager des nuances plus à même de rencontrer la nature propre du visuel :

La logique de la prédication a une double valeur : elle ne connaît que le oui et le non. Elle n'a pas accès à l'indéterminé, au potentiel, à l'absent ou au néant. « Rien » n'a pas de prédicat. Or, sans le divers, le polysémique, le sensuel et le polyvalent, l'on ne saurait réfléchir véritablement sur les images ${ }^{1}$.

Comment fonctionne donc cette logique de la différenciation, cette logique des contrastes qui semble - aux yeux de G. Boehm — définir la mécanique propre aux images ? Boehm prend comme exemple une toile très célèbre, $L a$ danse (1909-1910) de Henri Matisse. Notre attention - dit-il — est automatiquement divisée entre deux plans : d'une part, celui des figures rouges, conçues sur un mode ornemental et, d'autre part, celui du fond dont elles se détachent. Or, le sujet de l'image n'est ni l'un ni l'autre mais « l'un dans l'autre ». Il y a une différenciation première entre les figures et le fond ; on perçoit dans un premier temps des figures dansantes devant un fond comme si ces deux plans pouvaient être pris isolément. Cette différenciation est capitale puisqu'elle entraîne, par effet de réaction, par défense, une sorte de fusion qui vient contrecarrer la division première de l'attention : tout à coup, le fond (bleu/vert) s'avance, " prend lui-même figure dans les champs intermédiaires vacants », investit les creux et interagit directement avec les danseuses. Il y a «échange entre les plans de l'image». La différence iconique, dans ce tableau comme dans d'autres, dynamise la perception : elle

${ }^{1}$ G. Boehm, «Par-delà le langage ? Remarques sur la logique des images », op. cit. 
permet « le passage d'un agencement figé, dans lequel le figuratif se trouve devant le contexte, à un processus d'interaction qui fait naître du sens». C'est cette différenciation qui «met en mouvement la production de sens iconique $»^{1}$.

Cette théorie nous apprend - selon Boehm - quelque chose de fondamental sur l'image : " toute image tire sa force de détermination de sa liaison avec l'indéterminé ». Autrement dit : nous rapportons automatiquement le représenté (les figures, les motifs) au contexte, au champ d'inscription, au fond à partir duquel il apparaît. Il y aurait une interaction, une « contamination visuelle » entre les plans de l'image rendue possible par la différenciation première entre deux réalités différentes. "La "différence iconique" présentifie une règle de la discrimination, du contraste visuel, qui recèle simultanément un voir-ensemble ». Seule cette dynamisation des plans nous rend apte à voir des images — c'est-à-dire des organismes énigmatiques qui sont autre chose que des objets (autre chose que des «états de fait matériels »). Seule cette interaction permet que « naisse ce surplus d'imaginaire » qui transcende l'entité strictement matérielle. Reste à savoir ce que serait une entité strictement matérielle puisque, potentiellement, la moindre tache peut faire l'objet de nos capacités imageantes. En conclusion, pour Boehm, la différence iconique serait strictement visuelle, c'est-à-dire indépendante du paradigme langagier; elle poserait les rudiments d'une grammaire non verbale du visuel.

\section{L'image montre la monstration}

La logique de la monstration, que Boehm reconnait dans toute expression visuelle, guide la théorie de la différence iconique. L'expérience de la gestualité permet de saisir en quoi la monstration corporelle implique ellemême une différenciation fondamentale : le corps peut ainsi être considéré comme le fond continu, "opaque et impénétrable», à partir duquel se détachent les gestes singuliers. Les gestes vont et viennent depuis le corps dans lequel ils sont fondés. La signification naît quelque part de cette différenciation entre corps et gestes, qui implique - on l'a compris - non seulement une différence oppositive entre fond et signes gestuels, mais aussi le « geste moteur de leur liaison $»^{2}$.

${ }^{1}$ G. Boehm, « Par-delà le langage ? Remarques sur la logique des images », op. cit.

${ }^{2}$ G. Boehm, «Ce qui se montre. De la différence iconique», op. cit., p. 40. Cf. Dürer, Jésus parmi les docteurs de la loi (1506). 
Un autre exemple nous permet de saisir la logique iconique décrite par G. Boehm. Une toile de Mark Rothko, N ${ }^{\circ}$ 7, 1960 (Sezon, Japon, Musée d'Art moderne) exemplifie idéalement, selon l'auteur de cette analyse, l'idée d'un «monde au sens muet», non discursif. Le titre lui-même traduirait « une réserve à l'égard de toute dicibilité », réserve caractéristique du peintre qui privilégie souvent le titre «Untitled». Qu'observe-t-on ici ? Un fond brun sombre sur lequel apparaissent quatre champs colorés réalisés en « lasures », c'est-à-dire en couches semi-transparentes. À partir du fond et de son opacité profonde, les champs de couleurs semi-transparents semblent émerger à des distances différentes: le rapport du fond et des champs colorés, et le rapport conséquent de l'opacité et de la transparence, crée donc un ensemble de relations dynamiques. Les zones de couleur donnent l'impression de se dégager du fond et d'y retomber sans cesse. Autrement dit, le tableau, et sa différence iconique, font en sorte que l'image soit perçue comme mouvante et signifiante : l'image est une "équation énergétique » qui permet que quelque chose se montre.

Le mutisme de Rothko va de pair avec le pathos et l'affect. Ses tableaux, vides à l'apparence, génèrent en effet une sémantique, ils donnent l'impression de respirer et cet agencement vertical des surfaces fait allusion à un corps qui (sans être humain) semble vivant ${ }^{1}$.

Pour éprouver la thèse de la différence iconique (pour voir si elle est généralisable), Boehm se demande s'il existe des images sans champ visuel, c'est-à-dire sans cet horizon indéterminé et non thématique à partir duquel, par effet d'écart, se développeraient ou se distingueraient des thèmes. Des images qui feraient obstacle à cette grammaire de base du visuel que Boehm identifie dans l'idée d'une différenciation constitutive de l'iconicité. Deux cas-limite émergent alors : celui des structures «all over » de Pollock et celui des peintures de camouflage militaires. Dans ces deux cas, on observe un nivellement de la structure différenciée fond-figure, une réduction de l'asymétrie au profit d'une « densité d'apparition », densité telle qu'en réalité plus rien n'apparaît. Or, selon Boehm, seule la structure fond-figure permet de saisir cette densité où la différence des plans parait effacée. Autrement dit, c'est bien parce que notre faculté perceptive cherche - face à l'image - la différence iconique, ou plutôt que cette différence iconique invite la perception à jouer le jeu de l'image, qu'une telle densité se révèle efficace. Celle-ci n'apparaît pas d'un coup : « Le "tout sans parties" que ces images se

${ }^{1}$ Ibid., p. 46. 
voulaient de fonder ne nous atteint pas comme un éclair, mais veut lui-même être expérimenté, appréhendé à travers un acte $»^{1}$. Les expériences all over de Pollock mettent en défaut la logique iconique tout en la faisant apparaître. De la même manière, l'expérience de la peinture de camouflage militaire révèle notre propension à produire du sens à partir d'une différenciation essentielle (cf. les peintures de camouflage d'Andy Warhol). Puisqu'elle est sans différenciation, elle laisse disparaître ce quelque chose qui se distinguait $\mathrm{du}$ fond pour un «all over» qui cache toute figure. On serait donc ici confronté au processus en négatif de la perception, se repliant sur l'opacité, faisant marche arrière et réintégrant le visible au fond dont il émerge (fond que Boehm appelle encore la « littéralité matérielle ») : "L'iconique s'y dissout dans un continuum des choses apparemment recréé. De telles images de camouflage servent en quelque sorte à aveugler le spectateur, à l'empêcher de voir, en cherchant justement à réduire autant que possible la différence iconique». Autrement dit: «niveler le contraste, c'est faire disparaître l'image : le phénomène du camouflage l'illustre bien $»^{2}$.

\section{Un affranchissement relatif}

Paradoxalement peut-être, en cherchant à défendre la spécificité des logiques propres aux images, en tentant de s'affranchir du paradigme langagier pour la compréhension du visuel, Gottfried Boehm assume lui-même la référence au modèle linguistique établi par Saussure. Comme Saussure avait montré que la signification des mots ne repose pas sur des similarités avec le monde mais provient de pures oppositions internes, G. Boehm veut défendre l'idée que de la même manière (et donc avec la même légitimité) — les images ne tirent pas leur sens de similarités avec le monde réel mais bien de purs jeux internes d'oppositions visuelles qui stimulent la perception et invitent à un dépassement : le «contraste visuel» accompagne la naissance du sens des images. Une image implique toujours un contraste, et il importe d'en établir la logique pour décrire les effets de sens qui se développent au cœur même de la perception. Même un tableau monochrome «tire son iconicité d'une différence », à savoir dans ce cas précis celle qui sépare le champ coloré d'un mur.

De l'aveu même de Boehm, la théorie de la Gestalt n'est pas pour rien dans l'établissement de cette théorie : «Une pensée de la différentiation fait

${ }^{1}$ Ibid., p. 33.

${ }^{2} I d$. 
donc appel non plus au mécanisme dialectique de la négation réciproque, mais plutôt à la distinction que la psychologie de la Gestalt a décrit comme une distinction en figure et en fond $»^{1}$. Dans les exemples célèbres des figures bistables du visage/vase ou du lapin/canard, plutôt que de se concentrer sur la logique oppositive, encore trop exclusive selon Boehm, il faudrait analyser ce qu'il se passe exactement pour le regard quand il bascule d'une perception à une autre. Ce qui intéresse le théoricien de l'image, ce n'est pas seulement le système d'opposition des signes, mais la manière dont l'image est opératrice de liaison. Ce qui semble nous ramener (dit Boehm) vers « le sens premier du mot logos: "legein", c'est encore la ligature, le lien $»^{2}$.

La théorie de la «différence iconique » intervient à différents endroits dans les textes de Boehm, et semble porter sur plusieurs niveaux - le premier niveau étant celui sur lequel on s'est attardé ici. (1) Elle vise l'opposition entre le support matériel et la signification (la « représentation » visuelle). Comme dit plus haut, dans les images, il y a toujours une différence grâce à laquelle un ou plusieurs thèmes sont reliés à un champ formel qui, lui, est non thématique. Certaines lignes peuvent tout à coup apparaître comme des figures, et se gorger de sens. Pour le dire autrement encore : « le corps matériel des images fait fond à l'émergence, au sein même d'un champ visuel qui se différencie, de quelque chose qui émergera en tant que ceci ou cela $»^{3}$. (2) Mais la théorie de la différenciation iconique se réfère encore plus largement à la différence entre la représentation artistique et son modèle dans la réalité (le référent), ou entre l'image et l'objet. À partir de quoi Boehm peut élaborer une critique de la mimèsis entendue comme stricte imitation d'une réalité sans contamination par l'imaginaire de l'artiste. Contrairement à ce qu'une théorie basique de l'imitation pourrait laisser supposer, réaliser une image revient à "procéder à un acte de différenciation » à même le matériau sensible ${ }^{4}$. $\mathrm{Il}$ y a une «tension foncière entre la littéralité matérielle et ce qui s'en détache comme présentation visuelle », aspects qui ne sont jamais séparés en droit $^{5}$.

De la matière naît du sens, parce que les valeurs visuelles réagissent les unes aux autres à travers l'acte d'observation. Cela est mis en mouvement par une

\footnotetext{
${ }^{1}$ Ibid., p. 35 . de sens.

${ }^{3}$ Ibid., p. 33.

${ }^{4}$ Ibid., p. 31.

${ }^{5}$ Ibid., p. 34.
}

${ }^{2}$ En ce sens, on ne voit plus très bien ce qui différencie grammaire et logique, comprises comme théorie des liaisons entre éléments a-signifiants, liaisons productrices 
asymétrie caractéristique entre la figuration et l'horizon indéterminé, dans laquelle s'édifient également toutes les autres qualités spécifiques à l'image : vie, temporalité, affect, espace, narration, etc. Bien que ce processus puisse tout à fait être décrit, il ne devient adéquatement accessible qu'à travers l'acte de l'observation. Il s'agit d'un sens non prédicatif, qui n'est précédé par nul logos langagier, et sur lequel viennent bien sûr se greffer tous les discours langagiers requis : iconologies ou interprétations. Les images ne représentent pas un royaume clos. Mais leur culture vit du fait qu'elles affirment, face au marmonnement incessant des discours et au bruit des débats, l'étrangeté qui les habite, leur dense mutisme et leur évidente plénitude. Par-delà le langage, il existe de puissants espaces de sens, d'insoupçonnés espaces de visualité, de tonalité, de gestes, de mimique et de mouvement. Ils n'ont nul besoin d'amélioration ou de justification après-coup par la parole. C'est que le logos n'est justement pas que la prédication, la verbalisation et le langage. Son environnement est significativement plus vaste. Il importe de le cultiver ${ }^{1}$.

Le rêve d'une "sortie de l'image » par l'exercice de la raison, porté, à la suite des travaux de Rorty, par les tenants du Linguistic Turn, cherchait selon Boehm à défaire le statut «énigmatique» de l'image à mi-chemin entre « chose et non-chose», entre "tangibilité et rêves éthérés». On a voulu refuser à l'image sa nature d' « irréalité réelle». Selon Gottfried Boehm, les détracteurs de l'image auraient trop souvent tenté de réduire l'image à l'une de ses composantes, de «dissoudre l'existence hybride de l'iconique » - en faisant de l'image soit une chose qui ne renverrait vers rien d'autre qu'ellemême (un simple objet), soit un élément strictement cognitif (sans " assise matérielle »). Or, l'échec des différentes tentatives visant à se débarrasser de l'imaginaire, c'est-à-dire à se débarrasser de l'iconique, montre à quel point ces composantes sont fondamentalement liées. Boehm prend l'exemple de l'art minimal : là où Frank Stella cherchait à démontrer la littéralité des sculptures ou des peintures minimalistes (What you see is what you see manière de " purifier la perception » de toute idéalisation), c'est-à-dire là où il défendait pour les œuvres minimalistes le statut d'objet simple et inexpressif, il a bien fallu reconnaître que « la force projective de la perception s'y enflammait également sur un mode iconique $»^{2}$. Ou pour le dire plus simplement : même un simple parallélépipède rectangle posé devant nous sur le sol éveille (" enflamme ») notre capacité à projeter du sens sur ce qu'on voit $^{3}$. Cette nature double de l'iconique - l'idée d'une tension fondamentale

${ }^{1}$ G. Boehm, « Par-delà le langage ? Remarques sur la logique des images », op. cit. ${ }^{2} I d$.

${ }^{3}$ Cf. les analyses de Didi-Huberman dans Ce que nous voyons, ce qui nous regarde, Paris, Minuit, 1992. 
entre la nature concrète de l'image, sa nature plastique, sa nature d'entité matérielle, et sa nature immatérielle (sa capacité à convoquer des contenus absents) amènera G. Boehm à élaborer sa théorie de la différence iconique : la logique qu'il s'agit de restituer est celle par laquelle un élément visible désigne/appelle quelque chose d'absent. On n'est pas si loin de la philosophie du symbolique et on sent bien par ailleurs l'inspiration phénoménologique d'une telle théorie (Merleau-Ponty, Sartre) : « Nulle image ne peut se défaire de ce décalage irrévocable, et aucune ne crée de la présence sans l'incontournable ombre de l'absence $»^{1}$.

\section{Conclusion}

En réalité, aujourd'hui le débat autour de la préséance du visuel sur le langagier (ou l'inverse) semble moins vif et partiellement dépassé : tout le monde s'accorde sur le fait qu'une opposition exclusive entre, d'une part, le pouvoir absolu du langage, auquel l'image serait asservie en tant que simple redoublement illustratif et, d'autre part, l'autonomie radicale du visuel (les images muettes pures de tout contact avec les mots) est stérile.

G. Didi-Huberman a animé un débat épistémologique semblable du côté francophone en proposant en 1990 (Devant l'image) de combiner les apports de la sémiologie et de la phénoménologie - et cette suggestion rencontre les propositions énoncées par les protagonistes de la Bildwissenschaft. L'idée s'impose avec évidence : étudier le(s) sens des œuvres (les discours qu'elles portent, les effets qu'elles provoquent) mais jamais indépendamment des conditions matérielles d'énonciation, jamais sans étudier comment, c'est-à-dire par quels moyens concrets, le sens s'incarne dans des formes/lignes/couleurs. Parallèlement, étudier les formes et la manière dont elles touchent nos sens mais jamais indépendamment de l'univers de signification qui s'ouvre avec l'œuvre.

On peut difficilement, à partir de la théorie de Gottfried Boehm, répondre définitivement à la question : Y a-t-il dans le domaine du visuel l'équivalent d'une grammaire (ensemble de principes formels qui structurent le sens) ? S'il défend avec conviction l'idée d'un élargissement du logos au registre visuel, Boehm écarte lui-même la possibilité de quelque chose qui fonctionnerait comme une grammaire. Il faut bien saisir au moins que la théorie de la différence iconique ne se réduit pas à une simple grammaire des contrastes. Au sens de Boehm, on l'a compris, le faire-image (l'iconicité ou

${ }^{1}$ G. Boehm, « Par-delà le langage ? Remarques sur la logique des images », op. cit. 
l'imagéité selon les traductions de Bildlichkeit) ne dépend pas exclusivement de l'objet représenté - autrement dit, l'image n'est pas un simple véhicule pour une signification déjà instituée. Le sens d'une image survient dans l'événement de la perception, événement dont il importe de livrer une description phénoménologique : «La logique des images ne peut se résumer à une grammaire iconique: elle implique des corps auxquels elles se montrent et par lesquels elles peuvent se montrer ${ }^{1}$ - autrement dit, l'image dépend intrinsèquement $\mathrm{du}$ spectateur (le corps regardant) et du médium (le corps matériel de l'image).

La problématique du tournant iconique, qui marquerait l'abandon du paradigme langagier pour l'interprétation des images (ou qui affirmerait en tout cas l'irréductibilité du champ visuel au paradigme langagier), s'est rapidement imposée au cœur des débats de la Bildwissenschaft. Fort de ses recherches sur la psychologie du XIX ${ }^{\mathrm{e}}$ siècle et sur l'image mentale ${ }^{2}$, le philosophe et théoricien des médias Klaus Sachs-Hombach, dans un esprit assez semblable à celui de Boehm, s'est refusé - convaincu de la fragilité de l'analogie entre image et texte - à simplement transposer la méthode d'analyse sémiotique aux représentations visuelles. Selon lui, il n'y aurait pas dans le domaine du visuel d'équivalent à la syntaxe du langage, en dépit de la possibilité — identifiée par l'analyse iconologique — de mettre au jour un vocabulaire des formes et des règles combinatoires. Les images seraient incapables d'activer toutes les fonctions complexes que certaines propositions linguistiques peuvent porter (par exemple pour le cas des énoncés illocutoires). En affirmant que les images ne possèdent pas des fonctions aussi complexes, Sachs-Hombach semble contribuer à renforcer l'idée selon laquelle la représentation visuelle serait plus pauvre que la proposition langagière. Mais, en réalité, il revendique plutôt une sous-détermination de l'image : en tant qu'artefacts matériels, les images dépendent des compétences perceptives du spectateur. Elles sont inachevées, du point de vue de leur signification, tant que le spectateur ne s'est pas engagé dans un processus de réception, le spectateur étant, en dernier recours, celui qui offre aux images des "significations visuelles », c'est-à-dire des significations en accord avec les éléments visuels (par lui) observés. Selon cette critique, la sémiotique serait en quelque sorte trop "mécanique» dans l'étude de la production du sens, inefficace à établir une pragmatique de l'interprétation

\footnotetext{
${ }^{1}$ G. Boehm, « Ce qui se montre. De la différence iconique », op. cit., p. 39.

${ }^{2}$ Voir les analyses éclairantes de M. Rampley, « Bildwissenschaft », dans T. Lenain, H. Loche, A. Pinotti, M. Rampley, C. Schoell-Glass, K. Zijlmans (éds. ), Art History and Visual Studies in Europe, op. cit., p. 122.
} 
des images, manquant trop souvent le fait que les images deviennent signifiantes dans des contextes particuliers et pas seulement sur la base d'un jeu interne entre des signifiants visuels. Comme on le perçoit, le problème est vaste, et la conception du langage mobilisée par ces arguments parfois un peu pauvre. On ne voit pas pourquoi la sous-détermination ou l'inachèvement, qui appellent un effort interprétatif réel de la part du récepteur, et qui requièrent une pragmatique de la réception, joueraient un rôle moindre dans le champ du langage. Par ailleurs, sans se plier pour autant à un langage propositionnel qui lui imposerait une lecture linéaire, la réception d'une œuvre visuelle peut certainement mobiliser différentes formes de langage et d'écriture, selon des modèles multiples de rapport image/texte. 